Einfluss d. Chlornatriums auf die chem. Zusammensetzung d. Gehirnes. 335

auch in den Fällen von Kochsalzeinspritzung, während in der Gesammtmasse der Hirnhemisphären die Schwankung unbeträchtlich ist, denn sie geschieht zwischen 0,482 und $0,4852 \%$.

Die Ursache davon liegt in der grösseren Energie des Stoffwechsels der Corticalis als der Gesammtmasse, welche an zelligen Elementen viel ärmer ist.

In der Gesammtmasse der Hemisphären wächst das Natrium vom Normalen $0,09 \%$ bis 0,32 und das Kalium nimmt von 0,39 bis $0,25 \mathrm{ab}$, und so bleibt die Summe beider Metalle $\mathrm{K}+\mathrm{Na}=0,48 \%$.

In der grauen Substanz gelangt man von den Normalzahlen 0,013 für das Natrium und 0,62 für Kalium zu 0,17 für das Natrium und 0,47 für das Kalium.

3. Die Procentmenge Chlor, welche im Gehirne enthalten ist, nimmt durch diese Injectionen zu, aber nur soviel, als die Zunahme an Natrium betrifft, zu deren Sättigung eine grössere Menge Chlor nothwendig ist als zum gleichen Gewichte Kalium.

Diese Zunahme steht im geraden Verhältnisse zu der des Natrium und erreicht das Doppelte des Normalen.

4. Die Injectionen von Chlornatrium, zu wiederholten Malen durch die Carotiden auf die Blutbahn des Gehirnes geübt, reichen aus, das venöse Blut hellroth zu färben und das Muskelgewebe mehrere Stunden nach dem Tode des Thieres und seines Nervensystems reizbar und contractil zu erhalten.

\title{
Ueber die motorischen Nerven der Blase.
}

Von

\section{F. Nawrocki und B. Skabitsehewsky.}

Hierzu Tafel VI.

$\mathrm{Budg} \mathrm{e}^{1}$ ) beobachtete an aetherisirten Kaninchen Contraction der Blase, als er das blossgelegte Ritekenmarek am 4. Lendenwir-

1) J. Budge. Ueber das Centrum genito-spinale des N. sympathicus. Virchow's Archiv XV. Bd, Berlin 1858 S. 115. 
bel, oder den Banchsympathicus am 5. Lendenwirbel elektrisch reizte.

Gianuzzil) sah an Hunden Contraction der Blase, wenn er Nerven reizte, die von der 3., 4, und 5. Sacralwurzel herstammen und an der Bildung des plexus hypogastricus Theil nehmen, so wie während der Reizung der Nervenfäden des Bauchsympathicus, die von den ganglia mesenterica nach dem plexus hypogastricus hingehen. Er fand ferner, dass man stets Contraction der Blase beobachtet, wenn man das Lendenmark an zwei Stellen reizt, nämlich am 3. und am 5. Lendenwirbel.

Die erste Stelle ibberträgt ihre Wirkung durch Nervenfäden, welche zunächst durch die ganglia mesenterica hindurchgehen, bevor sie sich in den plexus hypogastricus hineinsenken; wenn man die betreffenden Nervenfäden durchschneidet, hat die Reizung des Ruickenmarkes am 3. Lendenwirbel keine Wirkung auf die Blase.

Die zweite Stelle (am 5 . Lendenwirbel) ibertrïgt ihre Wirkung durch Sacralfäden, die in den plexus hypogastricus direct iibergehen.

Budge $\mathrm{e}^{2}$ ) stellte Versuche hauptsiichlich an jungen Hunden an. Er setzte eine Wassersäule mit der Blase in Verbindung; auf diese Weise konnte er an dem durch die Muskelcontraction hervorgebrachten Wasserdrueke den Grad der Contraction messen. $\mathrm{Er}$ hatte diese Verfahrungsweise in zweierlei Weise angewandt. Entweder machte er nämlich am Vertex der Blase einen Einschnitt und band eine graduirte Glastöhre ein, unterband die Harnoöhrenmündung and fülte die Blase und bis zu einer gewissen Höhe die Glasröhre mit Wasser. Oder er brachte einen mit einer doppelt gebogenen graduirten Glasröhre verbundenen Katheter durch die Harnröhre in die Blase, schnürte die Harnröhre um den Katheter zu und fiullte die Blase mit Wasser. Aus seinen Untersuchungen ging hervor, dass: „durch die vorderen Wurzeln der 3 . und 4.

1) J. Gianuzzi. Note sur les nerfs moteurs de la vessie. Comptes rendus hebdomadaires des séances de l'Academie des sciences. T. LVI. Paris 1863. Janvier p. 53.

2) J. Budge. Ueber den Kinfluss des Nervensystems auf die Bewegung der Blase. J. Henle's und C. v. Pfeufer's Zeitschrift für rationelle Medicin 1864. Dritte Reihe. XXI. Bd. S. 1 und 174. 
Kreuzbeinnerven, nicht aber durch die vorderen Wurzeln der 1. und 2. Kreuzbeinnerven motorische Nerven zur Blase hingehen; dass es im Ruickenmarke eine ausgezeichnete Stelle gibt, welehe von den unteren Lendenwirbeln eingeschlossen ist, an welcher Reizung fast constant Bewegung der Blase zur Folge hat, und die Erregbarkeit viel länger besteht, als an allen tubrigen Stellen des Ruickenmarkes."

Die Resultate seiner Untersuchungen fasst $B u d g e$ in folgendem Satze zusammen: „Es gibt also zweierlei Züge von motorischen Nervenfasern für die Muskeln der Blase, der eine ist in den vorderen Wurzeln des 3. und 4. Sacralnerven, der andere in dem plexus hypogastricus enthalten." Budge ${ }^{1}$ ) kommt bei seinen neuen Untersuchungen zu wesentlich anderen Resultaten. Er sagt: „Im plexus hypogastricus liegen nur sensible Fasern und keine motorischen. Die letzteren finden sich lediglich im ersten, zweiten und dritten Sacralnerven."

Sokow nin ${ }^{2}$ ) stellte seine Versuche hauptsächlich an Katzen an. Er beobachtete die Contractionen der Blase unmittelbar mit dem Auge.

Nachdem Sokownin sich iiberzengt hatte, dass die Reizung sowohl der Sacralwurzeln, als auch der sympathischen Nervenfäden (zum plexus hypogastricus) Contractionen der Blase hervorruft, reizte er die oberen Theile des Riickenmarks nach vorgängiger Durchschneidung aller Sacralwurzeln oder des Ruckenmarks im Niveau des 7. Lendenwirbels. Die Reizung des Rückenmarkes am Halse rief noch in diesem Falle deutliche, wenn auch schwächere Contractionen der Blase hervor, die nach Durchschneidung beider Nervenfäden, welche vom ganglion mesentericum inferius zum plexus hypogastricus hinziehen, nicht mehr zum Vorschein kamen. Die motorischen Erregungen (Impulse) des Gehirns erreichen also die Blase nicht nur auf dem Wege der Sacralnerven, sondern auch rermittelst des sympathischen Nervensystems (ganglion mesentericum inferius).

1) J. Budge. Zur Physiologie des Blasensehliessmuskels. Pf I üger's Archiv für Physiologie 1872. Band VI. S. 306.

2) Sokownin. Materialien zur Physiologie der Entleernng und Zu. rückhaltung des Harnes. Kasan 1877 (russisch). Referirt in Hofmann's und Schwalbe's Jahresberichte über die Fortschritte der Anatomie und Physiologie. Bd. VI. Abth. III. S. 87. 
H. Nussbaum ${ }^{1}$ ) fand an Katzen, dass: 1) die motorisehen Rückenmarksnerven der Blase zum Theil in der 1, hauptsächlich in der 2. und 3. vorderen Sacralwurzel verlaufen; 2) dass die motorischen sympathischen Nerven der Blase aus dem Rïckenmark unterhalb des 3. Lendenwirbels heraustreten und die Blase erreichen: a) vermittelst des abdominalen Grenzstranges, feiner grauer Nervenfädchen, ganglion mesentericum inferius, zweier breiten grauen Fäden, b) ausserhalb des ganglion mesenterium inferius vermittelst des Grenzstranges.

Während der Reizung des peripheren Abschnittes des Grenzstranges unterhalb des 5 . Lendenwirbels erhielt $\mathrm{Nussb}$ a u Contraction der Blase.

Dieser kurze Abriss der Litteratur zeigt, dass noch manche Widersprïche in den Angaben der Autoren iber den Verlauf der motorischen Nerven der Blase existiren, und dass weitere Versuche erwiunscht waren.

Unsere Versuche wurden hauptsichlich an Katzen (zum Theil an Kaninchen) ausgefülurt. Zunächst präparirten wir an einer grossen Anzahl von Thieren die an die Blasenwand herantretenden Nerven. Dieselben grelangen an die Blase ausschliesslich von der Seite ihres Halses, wo sie sich durchflechten und den plexus vesicalis bilden. Dieses Geflecht ist die Fortsetzung eines zweiten dickeren plexus hypogastricus, welches aus Nervenfäden und kleinen Ganglien besteht, die im lockeren Bindegewebe an den Seiten des mittleren Theiles des Rectums liegen. Die stärkeren Nerven. stämme dieses plexus hypogastricus haben eine quere Richtung zur Liängsachse des Rectums; ein Theil derselben endigt in den Wänden des Rectums, der andere geht weiter und erreieht die Muskelschichten der Urethra und der Blase. Die Ganglien im plexus hypogastricus heben sich deutlich hervor and sind bei schwacher Vergrösserung sichtbar; ibre Anzahl ist verschieden, annäherungsweise 20-30 auf jeder Seite.

Der plexus hypogastricus wird durch Zerspaltung zweier

1) H. Nussbaum. Zur Frage über die Innervation des m. detruxor. Arbeiten der Laboratorien der medicinischen Facultät in Warschau. Herausgegeben von F. Nawrocki. Bd. V. 1879. S. 120 (russisch). Referirt in Hoffmann's und Schwalbe's Jahresberichte. VIII. Bd. II. Abth. 1879. S. (i1. 
Arten von Nerven gebildet: die einen stammen von Sacralnerven $a b$, die anderen vom ganglion mesentericum inferius. Die ersten führen den Namen der Nervi sacrales und bestehen nur aus zwei Fäden; der eine geht vom 2., der andere vom 3. Sacralnerven ab. Diese beiden Nervenfäden liegen anfangs nahe aneinander, gehen jedoch, vor ibrer Zerspaltung in die feinen Fasern des plexus hypogastricus, weit auseinander. Ausser diesen zwei Nervenfäden hat der plexus hypogastricus $\mathrm{k}$ ein e anderen Verbindungen mit den Sacralnerven; in der Beckenhöhle erhält also die Blase ihre Nerven unmittelbar aus dem Rückenmarke durch die Sacralnerven. Eine unmittelbare Verbindung des plexus hypogastricus mit dem 1. Sacralnerven (cf. Sokownin l. c.) konnten wir nie constatiren.

Was nun die andere Art von Nerven anbelangt, d. b. diejenigen, welche vom ganglion mesentericum inferius zum plexus hy pogastricus herabsteigen, haben wir nach $\mathrm{K} \mathrm{ra} \mathrm{u} \mathrm{s} \mathrm{e}^{1}$ ) kurzweg als Nervi hypogastrici bezeichnet; sie steigen in die Beckenhöhle herab, beiderseits an der Seite des Mastdarmes und gelangen anf diese Weise in den rechten und den linken plexus hypogastricus. Diese Nerven bestehen aus grauen Nervenfasern und liegen in Gestalt zweier ziemlich dicker Nervenstämme hinter dem Bauchfelle. Beim Kaninchen geht vom ganglion mesentericum inferius zunächst ein gemeinsamer Nervenstamm ab, der im weiteren Verlaufe sich in zwei Nervenfäden theilt, die an beiden Seiten des Mastdarmes in den plexus bypogastricus sich hineinsenken.

Das ganglion mesentericum inferius, von dem die beiden Nervi hypogastrici ihren Anfang nehmen, liegt im Winkel an dem Orte, wo die arteria mesenterica inferior aus der aorta abdominalis entspringt. Bei Kaninchen ist das ganglion mesentericum inferius einfach, bei Katzen besteht dasselbe aus vier kleinen Ganglien, die untereinander durch dünne Nervenfäden verbunden sind, welche die arteria mesenterica inferior umringen. Zur Auffindung dieser Gauglien ist es also zweckmässig, zunächst den Anfang der arteria mesenterica inferior anfzusuchen.

Vom ganglion mesentericum inferius gehen ausser den Nervi hypogastrici noch folgende Nervenfüden ab: 1) je drei Aeste beiderseits zur pars lumbaris des Grenzstranges, wir bezeichnen dieselben als Nervi mesenterici: superior, medius, inferior; 2) ein Ast,

7) W. Krause. Die Anatomie des Kaninehens. Leipzig 1868, S. 268 . 
der die arteria mesenterica inferior begleitet, nervus mesentericus arteriae; 3) zwei feine Nervenfäden, die an das ganglion mesentericum superius bingehen, welches seinerseits dureh zahlreiche Nervenfäden mit dem ganglion coeliacum verbunden ist.

Als eine Anomalie, die jedoch hei Katzen ziemlich oft vorhanden ist, dürfen wir bezeichnen, dass vom ganglion mesentericum inferius statt drei nur zwei Nervi mesenterici abgehen, die entweder als zwei, oder wenn der eine im weiteren Verlaufe in zwei sich zerspaltet, in drei besonderen Fäden den Grenzstrang erreichen. Die Nervi mesenteriei liegen auf der Banchaorta, umgeben dieselbe und geben in den Grenzstrang ïber in der Höhe des 4.-6. Lendenwirbels. Die rechten Nervi mesenterici ziehen zum Grenzstrang zwischen Aorta abdominalis und Vena cava inferior. Der Grenzstrang hat keine dire ete Verbindung mit dem plexus hypogastricus, wie die Abbildung deutlich zeigt.

Was nun den sichtbaren Ursprung der Sacralnerven aus dem Riickenmarke anbetrifft, so sind meine Untersuchungen in vollem Einklange mit den Angaben Sokownin's, nämlich: die 1. Sacralwurzel beginnt entsprechend dem unteren Drittel des 5 . Lendenwirbels, die 2. an der Grenze des oberen und des mittleren Drittels des 6. Lendenwirbels, und die 3. am unteren Drittel desselben Wirbels.

Aus dieser anatomischen Beschreibung geht hervor, dass die Blase mit dem Rüickenmarke durch zwei Nervenwege verbunden ist: der eine, obere, geht dureh das ganglion mesentericum inferius; den zweiten, unteren, in der Höhle des kleinen Beckens, bilden die Sacralnerven,

Um den störenden Einfluss der Contraction der Becken- und Bauchmuskeln zu eliminiren, haben wir unsere Versuche stets an curaresirten Thieren angestellt. Die Contractionen der Blase wurden mit dem blossen Auge beobachtet; wir haben uns überzeugt, dass das Einschneiden der Blasenspitze behufs Einbindung einer Canüle, so wie das Einführen eines Katheters stets Complicationen mit sich bringen, die die Reinheit der Beobachtung illusoriseh machen; es werden ja in beiden Fällen die sensiblen Nerven der Blase und im zweiten auch die der Urethra gereizt, was doch bei diesen Versuchen nicht gleichgilltig sein kann. Aus unserer zweiten Abhandlung wird der Leser ersehen, dass selbst nach geringfligigen Reizungen sensibler Nerven Blasencontractionen auf re- 
flectorischem Wege zu Stande kommen können. Es ist ausserdem sehr leicht die partiellen, durch Abkühlung oder Austrocknung bedingten Contractionen der Blase von der durch Nervenreizung hervorgerufenen totalen Contraction der Blasenmusculatur zu unterscheiden. Die ersteren sind schwach, unregelmässig wellenförmig, es kommt nie zur Runzelung der Blase noch zur Entleerung des Harnes. Solche störende Eigenbewegungen der Blase werden mitunter an Katzen, öfters an Kaninchen (deren Blase viel reizbarer ist, als die der Katze) beobachtet, und in solchen Fällen bleibt nichts tubrig, als den Versuch aufaugeben.

Während der Ruhe hat bei der Katze die Blase eine glatte, glänzende Oberfäche, hier und dort sieht man nur kleine Falten. Während der Reizung der an die Blase herantretenden motorischen Nerven, z. B. der Nervi hypogastrici, sieht man, dass, nach einer kurzen Zeit, die Blase sich schnell, beinahe augenblicklich runzelt unter Bildung zahlreicher Falten und sich im Ganzen contrahirt, wobei sie die Gestalt einer kleinen Wallnuss annimmt. Bei einer solchen Contraction erhebt sich die Spitze der Blase, ihre Längsachse wird senkrecht zur Achse der Wirbelsäule, der Inhalt der Blase wird wenigstens auf ein Drittel reducirt; mitunter werden diese Contractionen so stark, dass der Harn im Strahle entleert wird. Es zeigte sich, dass eine mittlere Füllang der Blase für derartige Beobachtungen am gainstigsten sei; aus diesem Grunde, falls die Blase prall angefullt war, haben wir durch leises Driicken auf die Bauchwand einen Theil des Harns vor Anstellung des Versuches entleert. Bei den Versuchen achtete man stets darauf, um die Blasenwand nicht zu berihren; denn die Blase reagirt sehr leicht auf mechanische Reize und kehrt nicht leicht in den erschlafften Zustand zurïck. Selbst starke Peristaltik des Rectums kann den Versuch stören. Die Hauptsache bei diesen Versuchen ist schnelle und vorsichtige Operation. Nachdem Curarelösung in die Vena jugularis externa eingespritzt und einige Zeit abgewartet wurde, bis unter klinstlicher Athmung das Thier völlig regungslos verblieb, wurde durch einen ergiebigen Schnitt in der Linea alba die Bauchhöhle eröffnet, die Darmschlingen vorsichtig bei Seite geschoben, die nöthigen Präparationen schnell vorgenommen, und sofort die Bauchböhle vermittelst Klemmpincetten wieder zugemacht. Die Beobachtung der Blasencontraction danert möglichst kurze Zeit, dagegen lange sind die Pausen, während welcher die 
Blase zugedeckt in der Bauchhöhle verbleibt. Uebrigens nach dem Tode des Thieres erhält sich die Erregbarkeit der Nervi hypogastrici gegen eine Stunde, die der Blase noch länger, so dass man, selbst unter diesen Umständen, manchen Versuch anstellen kann, wenn nur die Blase recht vorsichtig behandelt wurde.

Zur Reizung des Nerven bedienten wir uns des Du BoisReymond'schen Schlittenapparates (die secundäre Spirale mit 5000 Windungen) in Verbindung mit einem Stöhre r'schen Elemente. Die relative Stärke des Stromes wurde in Millimetern des Rollenabstandes ausgedrückt. Jedesmal nach dem Versuche wurde die Section vorgenommen und die Nerven der Blase rein präparirt.

Wir haben in dieser Richtung über einhundert Versuche angestellt; da jedoch in allen solchen Fällen, in denen keine unerwünschten Nebenumstände die Reinheit der Resultate markirten, wir stets dasselbe beobachteten, so beschränken wir uns auf die Mittheilung einiger Versuche aus jeder unserer Versuchsgruppen.

\section{Versuche.}

A. Reizung des Rückenmarkeśs.

I. 9/V. 88. Bei einer curaresirten Katze wurden vermittelst eines kleinen Trepans zwei Löcher im Rückgrat ausgebohrt, und zwar das eine am 1. und das zweite am 3. Halswirbel, und durch diese löcher zwei Nadel. elektroden ins Rückenmark eingestochen.

$11 \mathrm{~h} .5 \mathrm{~m}$. Reizung des Rückenmarkes bei $100 \mathrm{~mm}$. Rollenabstand (R. A.) gab starke Contraction der Blase.

" $40 \mathrm{~m}$. Reizung bei $100 \mathrm{~mm}$ R. A. starke Contraction.

" $45 \mathrm{~m}$. " " 70 " " " " $12 \mathrm{~h}$.

II. 11./V. 88. Bei einer curaresirten Katze wurde das Rückenmark am 1. Halswirbel durchschnitten. Die Nadelelectroden wurden ober- und nnterhalb des 2. Halswirbels eingestochen.

$11 \mathrm{~h} .30 \mathrm{~m}$. Reizung bei $100 \mathrm{~mm}$ R. A. starke Contraction.

, $40 \mathrm{~m}$.

$12 \mathrm{~h}$,

$\Rightarrow \quad 20 \mathrm{~m}$

" $30 \mathrm{~m}$

, $40 \mathrm{~m}$

$1 \mathrm{~b}$. .

\begin{tabular}{|c|c|c|}
\hline 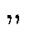 & $"$ & $"$ \\
\hline$"$ & " & $"$ \\
\hline$"$ & " & $"$ \\
\hline$"$ & $"$ & $"$ \\
\hline$"$ & $"$ & $"$ \\
\hline 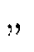 & $"$ & $70 \mathrm{~mm}$ \\
\hline
\end{tabular}


III. 27./XI. 89. Bei einem curaresirten Kaninchen wurden Nadelelectroden am 1. und 3. Halswirbel ins Rückenmark eingestochen.

$11 \mathrm{~h} .10 \mathrm{~m}$. Reiz. des Rückenmarkes bei $100 \mathrm{~mm}$ R. A. Contraction.

" $25 \mathrm{~m} . \quad$ Es wurde der N. Hypogastrious durchschnitten.

" $40 \mathrm{~m}$. Reiz. des Rückenmarkes bei $90 \mathrm{~mm}$ Contraction.

Es wurde das Rückenmark am 6. Lendenwirbel durchschnitten.

" $55 \mathrm{~m}$. Reiz. des Rückenmarkes bei $80-60 \mathrm{~mm}$ R. A. Ruhe.

\section{B. Reizung der 4. und 5. vorderen Lendenwurzel.}

IV. 23./11. 88. Bei einer curaresirten Katze wurden die 3., 4. und 5 . vordere Lendenwurzel freigelegt und durchschnitten. Man reizte die peripherischen Abschnitte mit Induktionsströmen.

1 h. $22 \mathrm{~m}$. Reizung der 5. Wurzel bei $100 \mathrm{~mm} \mathrm{R.A.} \mathrm{-} \mathrm{starke} \mathrm{Contraction.}$

$25 \mathrm{~m} . \quad, \quad, 4 . \quad, \quad, \quad$,

$28 \mathrm{~m} . \quad$ " $\quad 3 . \quad, \quad$ ",$\quad$ Rube.

V. 28./III. 88. Bei einer curaresirten Katze wurden die peripherischen Abschnitte der 3., 4., 5. und 6 . vorderen Lendenwurzel mit Induktionsströmen gereizt.

$11 \mathrm{~h} .50 \mathrm{~m}$. Reizung der 4 . Wurzel bei $110 \mathrm{~mm}$ R. A. starke Contraction.

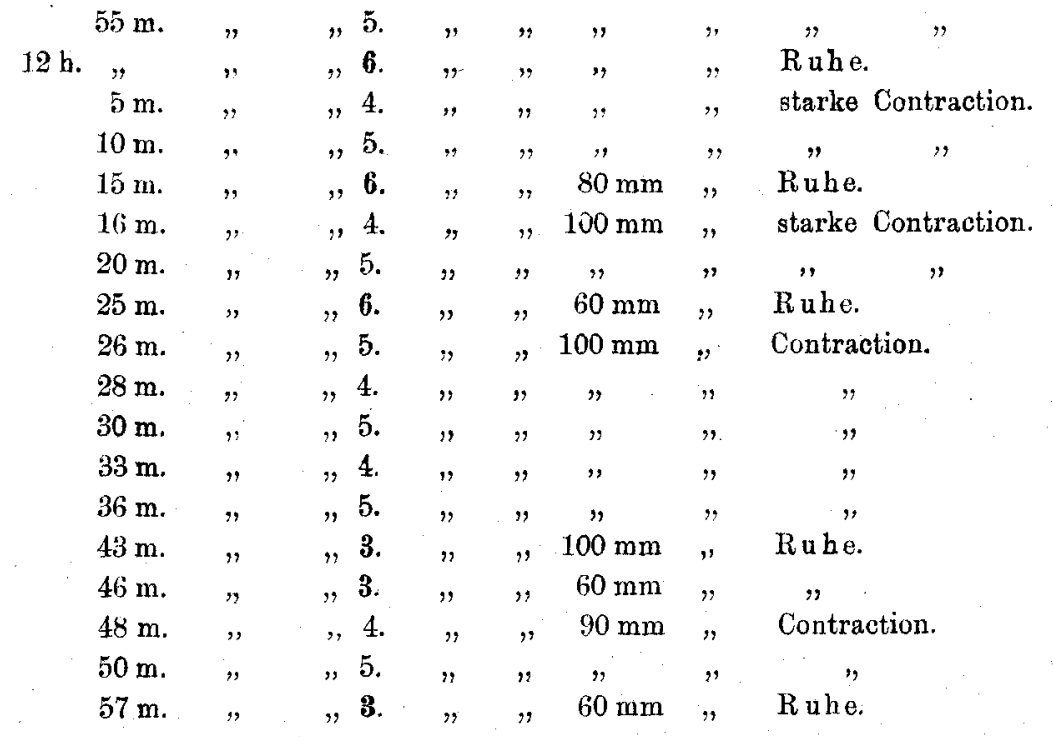

VI. 29./III. 88. Bei einer curaresirten Katze wurden die 3., 4., 5. und 6. vordere Lendenwurzel freigelegt und durchschnitten. Man reizte die peripherischen Abschnitte dieser Wurzeln mit Induktionsströmen. 
$12 \mathrm{~h}$. Reizung der 3. Wurzel bei $60 \mathrm{~mm} \mathrm{R}$. A. Ruhe.

\begin{tabular}{|c|c|c|c|c|c|c|c|}
\hline $5 \mathrm{~m}$. & $"$ & , 4. & $"$ & $"$ & $100 \mathrm{~mm}$ & $"$ & Contraction. \\
\hline $10 \mathrm{~m}$. & $"$ & $" 5$. & $"$ & $"$ & " & $"$ & starke Contraction. \\
\hline $15 \mathrm{~m}$. & $"$ &, 6. & $"$ & $"$ & $60 \mathrm{~mm}$ & , & Ruhe. \\
\hline $20 \mathrm{~m}$. & $\%$ & , 4. & $"$ & $"$ & $100 \mathrm{~mm}$ & $"$ & Contraction. \\
\hline $25 \mathrm{~m}$. & " & $" 5$. & $"$ & $"$ & $"$ & $"$ & $"$ \\
\hline $30 \mathrm{~m}$. & $"$ & $" 4$. & " & 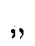 & $90 \mathrm{~mm}$ & $"$ & $"$ \\
\hline $35 \mathrm{~m}$. & $"$ & $" 5$. & $"$ & " & " & $"$ & " \\
\hline $40 \mathrm{~m}$. & $"$ & $" 4$. & $"$ & " & $80 \mathrm{~mm}$ & , & " \\
\hline $45 \mathrm{~m}$. & , & $" 5$. & $"$ & $"$ & " & $"$ & , \\
\hline
\end{tabular}

VII. 26./X. 88. Bei einer kleinen curaresirten Katze wurde der periphere Abschnitt der 4. vorderen Lendenwurzel mit Induktionsströmen gereizt.

11 h. $10 \mathrm{~m}$. Reizung der 4. Wurzel bei $100 \mathrm{~mm}$ R. A. starke Contraction.

$30 \mathrm{~m}$ $" 4$

$4 . \quad, \quad$, Contraction.

Es wurden beide Nn. Hypogastrici durchschnitten.

$35 \mathrm{~m}$. Reizung der 4 . Wurzel bei $100 \mathrm{~mm}$ R. A. Ruhe.

$40 \mathrm{~m} . \quad$ " , 4. " " $80 \mathrm{~mm} \quad, \quad$ "

VIII. 29./X. 88. Bei einer curaresirten Katze wurden auf beiden Seiten die 3., 4., 5., 6. und 7., vordere Lendenwurzeln freigelegt und durchschnitten. Man reizte die peripherischen Enden dieser Wurzeln mit Induktionsströmen.

$1 \mathrm{~h}$.

\begin{tabular}{|c|c|c|c|c|c|c|c|c|}
\hline $2 \mathrm{~m}$ & $"$ & ", & 6. , & $"$ & $"$ & $"$ & ", & Ruhe. \\
\hline $3 \mathrm{~m}$ & " & $"$ & 7. & $"$ & "' & $"$ & " & $"$ \\
\hline $5 \mathrm{~m}$. & $"$ & $"$ & 4. $\quad$, & $"$ & , & $"$ & $"$ & starke Contract. \\
\hline $6 \mathrm{~m}$. & $"$ & $"$ & 4. rechten & $"$ & $"$ & $"$ & $"$ & " \\
\hline $8 \mathrm{~m}$. & " & , & 5. linken & $"$ & $"$ & $90 \mathrm{~mm}$ & , & Contraction. \\
\hline $10 \mathrm{~m}$ & $"$ & $"$ & 5. rechten & , & $"$ & $"$ & $"$ & $"$ \\
\hline $20 \mathrm{~m}$. & , & , & 3. linken & $"$ & $"$ & $"$ & , & Ruhe. \\
\hline $21 \mathrm{~m}$. & $"$ & $"$ & 3. rechten & $"$ & $"$ & $"$ & ", & ", \\
\hline $22 \mathrm{~m}$. & $"$ & ," & 4. linken & $"$ & ", & $"$ & $"$ & Contraction, \\
\hline $24 \mathrm{~m}$. & $"$ & " & 4. rechten & $"$ & $"$ & $"$ & $"$ & " \\
\hline $26 \mathrm{~m}$. & $"$ & $"$ & 5. linken & " & $"$ & $"$ & $"$ & $"$ \\
\hline $28 \mathrm{~m}$. & $"$ & $"$ & 5. rechten & $\eta$ & $"$ & $\%$ & , & $"$ \\
\hline $30 \mathrm{~m}$. & $"$ & $"$ & 6. linken & " & $"$ & $80 \mathrm{~mm}$ & $"$ & Ruhe. \\
\hline $31 \mathrm{~m}$. & $"$ & $"$ & $5 . \quad$, & $"$ & $"$ & $90 \mathrm{~mm}$ & ." & Contraction. \\
\hline $32 \mathrm{~m}$ & $"$ & $"$ & 5. rechten & $"$ & $"$ & $"$ & " & $"$ \\
\hline $34 \mathrm{~m}$. & " & $"$ & 4. linken & $"$ & $"$ & $"$ & $"$ & "' \\
\hline $36 \mathrm{~m}$. & $"$ & $"$ & 4. rechten & $"$ & $"$ & $80 \mathrm{~mm}$ & $"$ & " \\
\hline $38 \mathrm{~m}$. & , & $"$ & 3. linken & $"$ & $"$ & " & $"$ & Ruhe. \\
\hline $39 \mathrm{~m}$. & $"$ & " & 3. rechten & , & $"$ & $"$ & , & " \\
\hline
\end{tabular}


$1 \mathrm{~h} .46 \mathrm{~m}$. Reizung der 4 . linken Wurzel bei $70 \mathrm{~mm}$ R. A. Ruhe.

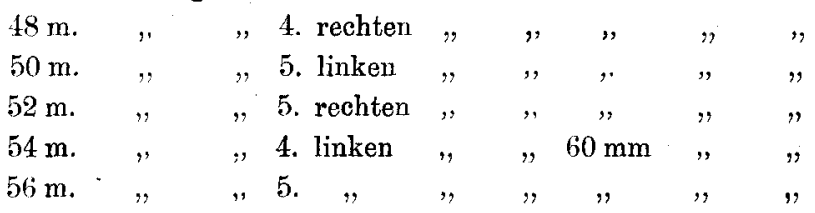

Es wurden die peripherischen Abschnitte der Nn. Hypogastrici gereizt.

$1 \mathrm{~h} .58 \mathrm{~m}$. Reizung des linken Hypogastricus bei $100 \mathrm{~mm}$ R. A. st. Contr. $2 \mathrm{~h} . \quad$ " rechten ", $, 100 \mathrm{~mm}, "$, ,

\section{Reizung des Lendentheiles des Grenzstranges.}

IX. 29./IX. 88. Bei einer curaresirten Katze wurde der linke Grenzstrang am 5 . Lendenwirbel durchschnitten und sein peripherer Abschnitt mit Induktionsströmen gereizt.

$12 \mathrm{~h} .15 \mathrm{~m}$. Reizung des Lendensympathicus bei $100 \mathrm{~mm} \mathrm{R}$. A. Contraction.

\begin{tabular}{|c|c|c|c|c|c|c|c|}
\hline $20 \mathrm{~m}$. & ", & $"$ & ", & $"$ & $90 \mathrm{~mm}$ & $"$ & $"$ \\
\hline $25 \mathrm{~m}$. & , & ", & $"$ & $"$ & " & $"$ & starke Contr. \\
\hline $30 \mathrm{~m}$. & , & $"$ & $"$ & $"$ & $\begin{array}{l}80 \mathrm{~mm} \\
70 \mathrm{~mm}\end{array}$ & $"$ & $\begin{array}{l}\text { Contraction. } \\
\text { starke Contr. }\end{array}$ \\
\hline
\end{tabular}

X. 21./V. 89. Bei einer grossen curaresirten Katze wurde der periphere Abschnitt des am 5. Lendenwirbel durchschnittenen linken Grenzstranges mit Inductionsströmen gereizt.

$11 \mathrm{~h}$. $5 \mathrm{~m}$. Reizung des Lendensympathicus bei $100 \mathrm{~mm}$ R. A. Contraction. $15 \mathrm{~m}$.

Es wurden beide Nn. Hypogastrici durchschnitten und zur Controlle, ob die Blasenmusculatur noch erregbar ist, mit Inductionsströmen gereizt (unmittelbar nach vorgenommener Reizung des Lendensympathicus).

$30 \mathrm{~m}$. Reiz. d. linken Lendensympath. bei $100 \mathrm{~mm}$ R. A. Ruhe.

$30 \mathrm{~m}$. " " rechten Hypogastricus , , " Contraction.

$40 \mathrm{~m}$. " " Lendensympath. " $80 \mathrm{~mm}$ " Ruhe.

$40 \mathrm{~m}$. " " " Hypogastricus , $100 \mathrm{~mm}$, Contraction.

$50 \mathrm{~m} . \quad "$ " " Lendensympath. ", $70 \mathrm{~mm}$, Ruhe.

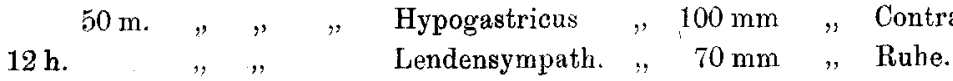

$12 \mathrm{~h} . \quad$ " " " Hypogastricus " $90 \mathrm{~mm}$, st. Contract.

$25 \mathrm{~m}$. " " Lendensympath. " $60 \mathrm{~mm}$ " Ruhe.

$25 \mathrm{~m}$. " " Hypogastrious " $100 \mathrm{~mm}$, st. Contract.

XI. 22./. 89. Bei einer grossen curaresirten Katze wurde das Rückenmark am 1. Halswirbel durchschnitten. Man reizte mit Induktionsströmen 
den unteren Abschnitt des am 5. Lendenwirbel durchschnittenen linken Grenzstranges.

$10 \mathrm{~h} .40 \mathrm{~m}$. Reiz. des Lendensympath. bei $100 \mathrm{~mm}$ R.A. Contraction.

$50 \mathrm{~m}$.

Man durchschnitt an beiden Seiten" die drei Nn. Mesenterici und die Nervenfäden, die vom oberen ganglion mesentericum an das untere herabsteigen; es wurde also das ganglion mesentericum inferius vom Grenzstrange vollkommen isolirt.

$11 \mathrm{~h} .15 \mathrm{~m}$. Reiz. des Lendensympath. bei $100 \mathrm{~mm}$ R. A. Ruhe.

$25 \mathrm{~m}$.

Es wurden beide Nn. Hypogastrici durchschnitten und der periphere Abschnitt des rechten mit Induktionsströmen gereizt, zur Controlle, ob die Blasenmusculatur noch erregbar geblieben ist.

$12 \mathrm{~h}$. $5 \mathrm{~m}$. Reiz. des Hypogastricus bei $100 \mathrm{~mm} \mathrm{R}$. A. Contraction.

$20 \mathrm{~m}$. " " Lendensympath., $80 \mathrm{~mm}$, Rulie.

$20 \mathrm{~m}$. " , Hypogastricus , , " Contraction.

XIr. 20./V. 89. Bei einem curaresirten Kaninchen wurde der linke Grenzstrang am 5. Lendenwirbel durchschnitten und sein peripherer Abschnitt mit Inductionsströmen gereizt.

$10 \mathrm{~h} .40 \mathrm{~m}$. Reiz. des Lendensympath. bei $100 \mathrm{~mm}$ R. A. starke Contract. $50 \mathrm{~m}$.

, , , " ,

, $150 \mathrm{~mm}$ " $200 \mathrm{~mm}$ " schwache ",

Man durchschnitt den $\mathrm{N}$. hypogastricus und reizte seinen peripheren Abschnitt mit Induktionsströmen, um die Reizbarkeit der Blasenmusculatur darzuthun.

$11 \mathrm{~h} .10 \mathrm{~m}$. Reiz. d. Hypogastricus bei $200 \mathrm{~mm}$ R. A. sehr starke Contr.

\begin{tabular}{|c|c|c|c|c|c|c|}
\hline $20 \mathrm{~m}$. & & & Lendensympath. & $\Rightarrow 150 \mathrm{~mm}$ & & Ruhe. \\
\hline $5 \mathrm{~m}$ & & " & $"$ & " $100 \mathrm{~mm}$ & & Ruhe. \\
\hline $\begin{array}{l}26 \mathrm{~m} . \\
35 \mathrm{~m} .\end{array}$ & & $"$ & $\begin{array}{l}\text { Hypogastricus } \\
\text { Lendensympath. }\end{array}$ & $\begin{array}{l}, \quad 300 \mathrm{~mm} \\
, \quad 80 \mathrm{~mm}\end{array}$ & ; ; & $\begin{array}{l}\text { starke Contraction. } \\
\text { Ruhe. }\end{array}$ \\
\hline $96 \mathrm{~m}$. & 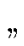 & $n$ & Hypogastricus & , $\quad 350 \mathrm{~mm}$ & " & Contraction \\
\hline $45 \mathrm{n}$ & " & " & Lendensympath. & $70 \mathrm{~mm}$ & "1 & Ruhe. \\
\hline $46 \mathrm{~m}$ & & , & Hypogastricus & , $300 \mathrm{~mm}$ & & starke Contraction. \\
\hline $5 \mathrm{~m}$. & & " & $\begin{array}{l}\text { Lendensympath. } \\
\text { Hypogastricus }\end{array}$ & $\begin{array}{l}\text { " } \quad 60 \mathrm{~mm} \\
\text { " } 250 \mathrm{~mm}\end{array}$ & 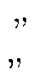 & $\begin{array}{l}\text { Ruhe. } \\
\text { starke Contraction. }\end{array}$ \\
\hline
\end{tabular}

XIII. 16./V. 89. Bei einem curaresirten Kaninchen wurde der periphere Abschnitt des N. Hypogastricus mit Induktionsströmen gereizt.

$10 \mathrm{~h} .30 \mathrm{~m}$. Reiz. des Hypogastricus bei $120 \mathrm{~mm}$ R. A. sehr starke Contr. $40 \mathrm{~m}$.

Es wurde der li in k Grenzstrang am 5. Lendenwirbel durchschnitten und sein peripherer Abschnitt, und unmittelbar darauf der des Hypogastricus electrisch gereint. 
10 h. $50 \mathrm{~m}$. Reiz. d. Lendensympath. bei $100 \mathrm{~mm}$ R. A. Ruhe.

\begin{tabular}{|c|c|c|c|c|c|}
\hline & $50 \mathrm{~m}$. & $"$ & & Hypogastricus & , $140 \mathrm{~mm}$ \\
\hline $11 \mathrm{~h}$. & & 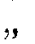 & & Lendensympath. & $90 \mathrm{~mm}$ \\
\hline $11 \mathrm{~h}$. & & $"$ & $"$ & Hypogastricus & $\Rightarrow 150 \mathrm{~mm}$ \\
\hline & $10 \mathrm{~m}$. & ,. & , & Lendensympath. & $80 \mathrm{~mm}$ \\
\hline & $10 \mathrm{~m}$. & " & $"$ & Hypogastricus & , $200 \mathrm{~mm}$ \\
\hline & $20 \mathrm{~m}$. & " & ", & $"$ &,$\quad 200 \mathrm{~mm}$ \\
\hline & $40 \mathrm{~m}$. & $"$ & " & $"$ & , $220 \mathrm{~mm}$ \\
\hline & $50 \mathrm{~m}$. & $"$ & , & Lendensympath. &,$\quad 70 \mathrm{~mm}$ \\
\hline & $50 \mathrm{~m}$. & $"$ & ", & Hypogastricus & , $250 \mathrm{~mm}$ \\
\hline $12 \mathrm{~h}$. & $15 \mathrm{~m}$. & ", & " & $"$ & ,, $300 \mathrm{~mm}$ \\
\hline
\end{tabular}
sehr starke Contr. Ruhe. sehr starke Contr. Ruhe. sehr starke Contr.

Ws wurde der Hypogastricus mechanisch (Zerquetschen mit einer Pincette) gereizt.

$20 \mathrm{~m}$. mech. Reiz. d. Hypogastricus R. A. Contraction.

$21 \mathrm{~m}$.

XIV, 29./IX. 90. Bei einer kleinen curaresirten Katze wurde auf beiden Seiten der Grenzstrang am 5. Lendenwirbel durchsclnitten, und die peripheren Enden dieser Nerven gereizt.

$12 \mathrm{~h} .55 \mathrm{~m}$. Reiz, d. rechten Lendensympath. bei $100 \mathrm{~mm}$ R.A. Contraction.

$1 \mathrm{~h} .5 \mathrm{~m} . \quad$, , linken

Beide Nn. Hypogastrici wurden durchschnitten.

$1 \mathrm{~h} .20 \mathrm{~m}$. Reiz. d. rechten Lendensympath. bei $50 \mathrm{~mm}$ R. A. Ruhe.

$30 \mathrm{~m}$. " ", linken , , , " , ,

Zum Nachweis, dass die Reizbarkeit der Blasenmusculatur erhalten war, wurden die peripheren Enden der Hypogastrici elektrisch gereizt.

$31 \mathrm{~m}$. Reiz. d, rechten Hypogastrici bei $100 \mathrm{~mm}$ R. A. Contraction.

$32 \mathrm{~m}$. " " " linken " " , , " "

$40 \mathrm{~m}$. " " "rechten " " , , , " ,

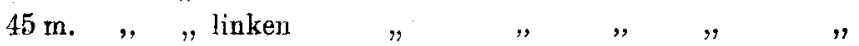

\section{Reizung der Nn. mesenterici (superior, medius, inferior).}

XV. 18./IV. 88. Bei einer curaresirten Katze wurden auf der linken Seite die drei vom Grenzstrange nach dem Ganglion mesentericum inferius abgehenden Nn. mesenterici durchschnitten und ihre peripheren Abschnitte elektrisch gereizt.

12 h. $50 \mathrm{~m}$. Reiz. d. Mesent. superior bei $110 \mathrm{~mm} \mathrm{R.A.} \mathrm{Contraction.}$

$1 \mathrm{~h}$.

$55 \mathrm{~m}$.

10

$30 \mathrm{~m}$. " " " $"$

$40 \mathrm{~m} . \quad, \quad, \quad, \quad$,

$\begin{array}{ccccc}\text { medius } & , & , & , & " \\ \text { inferior } & , & , & , & " \\ " n & , & 100 \mathrm{~mm} & , & , \\ \text { medius } & , & , & , & ., \\ \text { superior }, & , & , & ,\end{array}$


XVI. 19./IV. 88. Bei einer curaresirten Katze wurden die voin Greuzstrange abgetrennten peripheren Enden der drei Nn. mesenterici mit Induktionsströmen gereizt.

$11 \mathrm{~h} .50 \mathrm{~m}$. Reiz. d. Mesent. inferior bei $100 \mathrm{~mm} \mathrm{R.A.} \mathrm{Contraction.}$

$12 \mathrm{~h}$.

\begin{tabular}{|c|c|c|c|c|c|c|c|c|}
\hline & " & $"$ & " & medius & $"$ & $"$ & $"$ & $"$ \\
\hline $10 \mathrm{~m}$ & $"$ & $"$ & $"$ & superior & $"$ & $"$ & $"$ & $"$ \\
\hline $15 \mathrm{~m}$. & $"$ & $"$ & ", & inferior & $n$ & " & $"$ & " \\
\hline $20 \mathrm{~m}$. & ", & $"$ & $"$ & medius & $"$ & 19 & $"$ & ", \\
\hline $30 \mathrm{~m}$ & $"$ & $"$ & $"$ & superior & $n$ & $"$ & $"$ & " \\
\hline $40 \mathrm{~m}$. & $"$ & $"$ & " & inferior & $"$ & $"$ & $"$ & $"$ \\
\hline $50 \mathrm{~m}$. & , & ," & $"$ & $\begin{array}{l}\text { medius } \\
\text { superior }\end{array}$ & ", & ", & $"$ & $"$ \\
\hline
\end{tabular}

XVII. 30./I. 88. Bei einer curaresirten Katze wurden die peripheren Abschnitte der drei Nn. mesenterici elektrisch gereizt.

12 h. $50 \mathrm{~m}$. Reiz. d. Mesent. inferior bei $120 \mathrm{~mm}$ R. A. starke Contraction 5 $1 \mathrm{~h}$. $53 \mathrm{~m}$.

$\begin{array}{lcc}\text { medius }, & " & " \\ \text { superior }, & , & " \\ \text { inferior " } & 110 \mathrm{~mm} & " \\ \text { medius ", } & " & " \\ \text { superior ", } & , & "\end{array}$
Contraction.

$3 \mathrm{~m} . \quad, \quad, \quad, "$,

$5 \mathrm{~m} . \quad, \quad, \quad$,

$9 \mathrm{~m} . \quad, \quad, \quad$, superior " , "

"
",

XVIII. 22./XI. 87. Bei einer curaresirten Katze wurden alle drei Nn. Mesenterici zusammen unterbunden, vom Grenzstrang getrennt, und ihr peripherer Abschnitt elektrisch gereizt.

$12 \mathrm{~h} 15 \mathrm{~m}$. Reiz. d. Nn. mesenterici bei $100 \mathrm{~mm}$ R. A. Contraction.

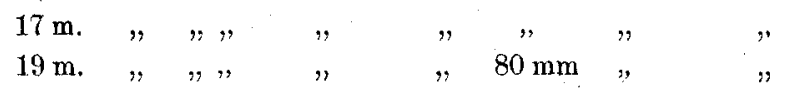

Es wurde der linke Hypogastricus durchschnitten.

$21 \mathrm{~m}$. Reiz. d. Nn. mesenterici bei $70 \mathrm{~mm} \mathrm{R.} \mathrm{A.} \mathrm{Contraction.}$

Es wurde der rechte Hypogastricus durchschnitten.

$23 \mathrm{~m}$. Reiz. der $\mathrm{Nn}$. mesenterici bei $60 \mathrm{~mm} \mathrm{R}$. A. Ruhe.

Um die erhaltene Reizbarkeit der Blasenmuskulatur darzuthus, wurden die peripheren Enden der Hypogastrici gereizt.

$24 \mathrm{~m}$. Reiz. d. Kinken Hypogastricus bei $100 \mathrm{~mm}$ R. A. Contraction.

$25 \mathrm{~m}$.

, rechten

XIX. 30./XI. 87. Bei einer curaresirten Katze wurden links alle drei Nn. mesenterici unterbunden, vom Grenzstrang abgeschnitten, und mit Induk* tionsströmen gereizt.

$12 \mathrm{~h}$. $5 \mathrm{~m}$. Reiz. der Nn. mesenterici bei $120 \mathrm{~mm} \mathrm{R}$. A. Contraction.

$10 \mathrm{~m}$.

$15 \mathrm{~m}$

$20 \mathrm{~m}$.

$25 \mathrm{~m}$. 
Man durchsohnitt beide Nn. Hypogastrici.

$30 \mathrm{~m}$. Reiz. der Nn. mesenterici bei $100 \mathrm{~mm}$ R. A. Ruhe.

Um die vorhandene Reizbarkeit der Blasenmusculatur darzulegen, wurde das periphere Ende des linken Nn. Hypogastricus gereizt.

$35 \mathrm{~m}$. Reiz. d. linken Hypogastricus bei $110 \mathrm{~mm} \mathrm{R}$. A. sehr st. Contr. $40 \mathrm{~m}$.

XX. 1./XII. 87. Bei einer curaresirten Katze wurden die drei Nn. mesenterici links unterbunden, vom Grenzstrang abgetrennt, und ihr peripherer Abschnitt elektrisch gereizt.

11 h. $10 \mathrm{~m}$. Reiz. d. Nn. Mesenterici bei $100 \mathrm{~mm}$ R. A. Contraction.

$15 \mathrm{~m}$.

", " " " " " ,

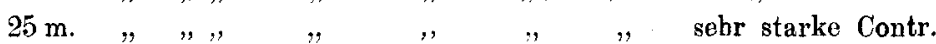

$30 \mathrm{~m}$.

Es wurden beide Nn. Hypogastrici durchschnitten.

$35 \mathrm{~m}$. Reiz. d. Nn. mesenterici bei $100 \mathrm{~mm}$ R. A. Ruhe.

Um die Reizbarkeit der Blasenmusculatur nachzuweisen, wurde das periphere Ende des linken Nn. Hypogastricus gereizt.

$36 \mathrm{~m}$. Reiz. d. Nn. Hypogastricus bei $100 \mathrm{~mm}$ R. A. sehr st. Contr.

$40 \mathrm{~m}$.

$50 \mathrm{~m}$.

XXI. 1./II. 88. Bei einer curaresirten Katze wurde der periphere Abschnitt des $\mathrm{Nn}$. mesentericus inferior elektrisch gereizt.

$12 \mathrm{~h} .10 \mathrm{~m}$. Reiz. d. N. mesentericus inferior bei $100 \mathrm{~mm} \mathrm{R}$. A. starke Contr. $13 \mathrm{~m}$.

Beide Nn. Hypogastrici wurden durchschnitten.

$12 \mathrm{~h} .16 \mathrm{~m}$. Reiz. d. N. mesent. infer. bei $100 \mathrm{~mm} \mathrm{R}$. A. Ruhe.

Die Reizung des peripheren Endes des linken Hypogastricus wies die Reizbarkeit der Harnblasenmusculatur nach.

$12 \mathrm{~h} .25 \mathrm{~m}$. Reiz. d. N. Hypogastricus bei $100 \mathrm{~mm}$ R. A. Contraction.

\section{E. Reizung der Nn. Hypogastrici.}

a) bei lebendem Thiere.

XXII. 1./1X. 87. Bei einer curaresirten Katze wurden beide Nn. Hypogastrici durchschnitten und ihre peripheren Enden elektrisch gereizt.

$10 \mathrm{~h} .28 \mathrm{~m}$. Reiz. des rechten Hypogastricus bei $120 \mathrm{~mm}$. R.-A. Contraction.

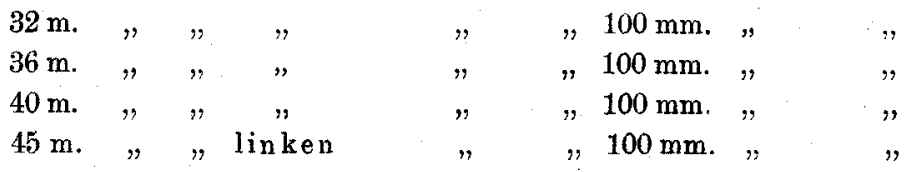

XXIII 17./IX. 87. Bei einer curaresirten Katze wurde der periphere Abschnitt der linken Hypogastricus gereizt. 
1 h. Reiz. des linken Hypogastricus bei $90 \mathrm{~mm}$ R.A. starke Contr.

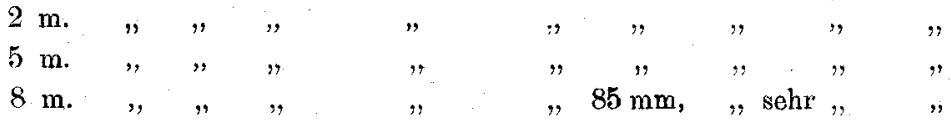

XXIV 23./XI. 89. Bei einem curaresirten Kaninchen wurde das periphere Ende des Hypogastricus elektrisch gereizt.

10 h. $30 \mathrm{~m}$. Reiz. des Hypog. bei $250 \mathrm{~mm}$ R. A. starke Contraction.
$40 \mathrm{~m}$.
Contraction.
$50 \mathrm{~m} . \quad, \quad, \quad " \quad, 250 \mathrm{~mm} \quad$ " starke Contraction.

11 h. Zerquetschung des Hypog. mit einer Pincette. Contraction.

b) nach dem Tode des Versuchsthieres.

XXV. 13./X. 88. Eine junge Katze starb sofort nach Injection der Curarelösung in die Iugularvene un $12 \mathrm{~h}$. - Die nach dem Versuche vorgenommene Section ergab einen Diaphragmabruch, beinahe der ganze Dünndarm lag in der Brusthöhle. - Es wurden die beiden Nn. Hypogastrici durchschnitten, und ihre peripheren Abschnitte mit Inductionsströmen gereizt.

12 h. $15 \mathrm{~m}$. Reiz. des linken Hypog. bei $100 \mathrm{~mm}$ R. A. Contraction.

$16 \mathrm{~m}$.

$17 \mathrm{~m}$. " "rechten

$22 \mathrm{~m}$. " " linken

$23 \mathrm{~m}$. " " rechten

$52 \mathrm{~m}$. " " , linken

$80 " \mathrm{~mm}$.
$"$
$"$

XXVI 23./V. 89. Eine schwangere Katze starb sofort nach Injection der Curarelösung in die Vena jugularis um $11 \mathrm{~h} .15 \mathrm{~m}$. Es wurden beide $\mathrm{Nn}$. Hypogastrici durchschnitten, und ihre peripheren Abschnitte elektrisch gereizt.

$11 \mathrm{~h} .30 \mathrm{~m}$. Reiz. des linken Hypog. bei $100 \mathrm{~mm}$ R. A. Contraction.

\begin{tabular}{|c|c|c|c|c|c|c|c|}
\hline $32 \mathrm{~m}$. & $"$ & $"$ rechten & 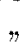 & $"$ & $"$ & $"$ & $"$ \\
\hline $37 \mathrm{~m}$ & $"$ & $"$ linken & " & $"$ & " & $n$ & $"$ \\
\hline $41 \mathrm{~m}$. & $"$ & $"$ rechten & $"$ & $"$ & $"$ & $"$ & starke Contraction. \\
\hline $50 \mathrm{~m}$. & $n$ & $"$ linken & $"$ & $n$ & $"$ & $n$ & Contraction. \\
\hline & $"$ & "rechten & $"$ & $"$ & $"$ & $"$ & $"$ \\
\hline $5 \mathrm{~m}$. & $"$ & $"$ linken & $"$ & $\eta$ & $"$ & $"$ & schwache Contr. \\
\hline
\end{tabular}

XXVII. 25./V. 89. Fine grosse Katze starb sofort nach Injection der Curarelösung in die Iugularvene um $10 \mathrm{~h} .50 \mathrm{~m}$. Es wurde der linke Grenzstrang am 5. Lendenwirbel durchschnitten, und sein peripherer Abschnitt elektrisch gereizt.

$11 \mathrm{~h} .10 \mathrm{~m}$. Reiz. des Lendensympathicus bei $80 \mathrm{~mm}$. R. A. starke Contr.

Es wurden beide Hypogastrici durchschnitten.

$20 \mathrm{~m}$. Reiz. des Lendensympathicus bei $80 \mathrm{~mm}$. R. A. Ruhe. 
Unmittelbar darauf wurden die peripheren Abschnitte der Hypogastrici elektrisch gereizt.

$20 \mathrm{~m}$. Reiz. des rechten Hypog. bei $80 \mathrm{~mm}$ R. A. sehr starke Contr.

\begin{tabular}{|c|c|c|c|c|c|c|c|}
\hline $20 \mathrm{~m}$. & $"$ & $"$ linken & $"$ & $"$ & , & $"$ & \\
\hline $5 \mathrm{~m}$. & , & $"$ & $"$ & $"$ & $"$ & $"$ & Contraction. \\
\hline $9 n$ & $n$ & $"$ rechten & $"$ & $"$ & $"$ & $n$ & schwache Contr. \\
\hline $\mathrm{m}$. & ” & $"$ linken & $"$ & $"$ & $"$ & $"$ & Contraction. \\
\hline$n$ & $n$ & $"$ & & $"$ & $"$ & $"$ & schwache Contr. \\
\hline & & $"$ & $\eta$ & " & $70 \mathrm{~mm}$. & $"$ & Ruhe. \\
\hline
\end{tabular}

Die unmittelbare Reizung der Blasenwand rief Contraction hervor.

XXVIII. 27./V. 89. Bei einem curaresirten Kaninchen wurde der periphere Abschnitt des N. Hypogastricus elektrisch gereizt.

11 h. $25 \mathrm{~m}$. Reiz. des Hypog. bei $100 \mathrm{~mm}$. R. A. Contraction.

Die künstliche Athmung wurde aufgehoben;

$30 \mathrm{~m}$. Tod des Thieres. Keine Herztöne, Carotis und Banchaorta pulsirten nicht mehr.

$45 \mathrm{~m}$. Reiz. des Hypog. bei $100 \mathrm{~mm}$ R. A. Contraction.

$12 \mathrm{~h}$.

\begin{tabular}{|c|c|c|c|c|c|c|c|c|}
\hline & $"$ & $n$ & $"$ & $n$ & $90 \mathrm{~mm}$ & $"$ & $"$ & \\
\hline $\mathrm{m}$. & $"$ & $n$ & $"$ & $"$ & $80 \mathrm{~mm}$ & $n$ & $"$ & \\
\hline $3 \mathbf{m}$. & $"$ & $n$ & n & $"$ & $\begin{array}{c}" \prime \\
70 \mathrm{~mm} .\end{array}$ & $n$ & schwache & Contraction. \\
\hline
\end{tabular}

\section{F. Reizung der 2. und 3, vorderen Sacralwurzeln.}

XXIX. 11./IV, 88. Bei einer curaresirten Katze wurden links die vorderen 1., 2, 3., 4. Sacralwurzeln und die 1. vordere Caudalwurzel vom Rückenmark getrennt; die peripheren Abschnitte genannter Wurneln wurden mit Induktionsströmen gereizt.

$11 \mathrm{~h} .55 \mathrm{~m}$. Reiz. der 3. Sacralis bei $100 \mathrm{~mm}$.

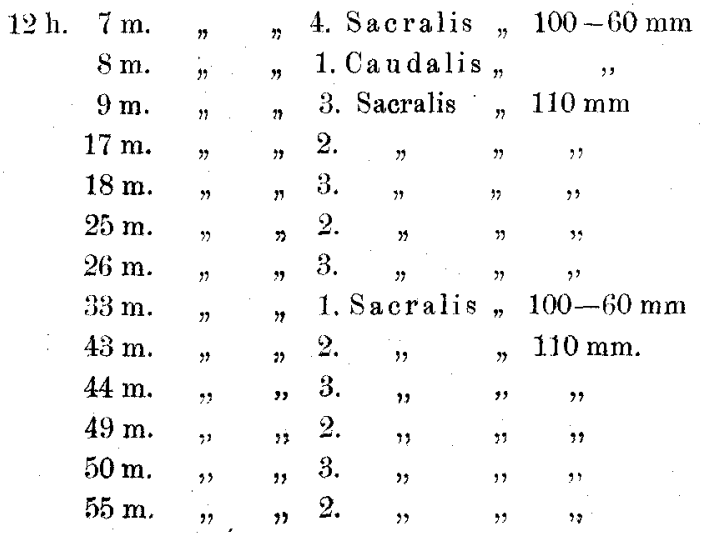

starke Contraction.

Ruhe.

Ruhe.

starke Contraction.

Contraction.

starke Contraction.

Contraction.

starke Contraction.

Ruhe.

Contraction.

starke Contraction.

Contraction.

starke Contraction.

Contraction.

XXX. 12./IV. 88. Bei einer curaresirten Katze wurden die 1., 2. und 
3. vorder en Sacralwurzeln vom Rückenmarke getrennt, und ihre peri pheren Abschnitte elektrisch gereizt.

$11 \mathrm{~h} .55 \mathrm{~m}$. Reiz. der 3. Sacralis bei $90 \mathrm{~mm}$ R. A. Contraction. $12 \mathrm{~h}$.

\begin{tabular}{|c|c|c|c|c|c|c|c|c|}
\hline & $"$ & $"$ & 2. & $"$ & $"$ & " & $"$ & $"$ \\
\hline $13 \mathrm{~m}$. & $"$ & , & 1. & Sacralis & , & $60 \mathrm{~mm}$. & , & Ruhe. \\
\hline $4 \mathrm{~m}$. & ", & " & 2. & , & $"$ & $90 \mathrm{~mm}$ & $"$ & Contraction. \\
\hline $20 \mathrm{~m}$. & $"$ & $"$ & 3. & $"$ & $"$ & " & $"$ & starke Contraction. \\
\hline $25 \mathrm{~m}$. & ; & ", & 2. & $"$ & $"$ & $"$ & $"$ & Contraction. \\
\hline $30 \mathrm{~m}$. & $"$ & $"$ & 3. & $"$ & $"$ & $"$ & $"$ & starke Contraction. \\
\hline $35 \mathrm{~m}$. & " & $"$ & 2. & $"$ & $"$ & $"$ & ", & Contraction. \\
\hline $40 \mathrm{~m}$. & $"$ & " & 3. & " & $"$ & $"$ & $"$ & $"$ \\
\hline $45 \mathrm{~m}$. & $"$ & $"$ & 2. & $"$ & $"$ & $80 \mathrm{~mm}$ & $"$ & " \\
\hline $50 \mathrm{~m}$. & $"$ & 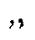 & 3. & $"$ & $"$ & , & $"$ & $"$ \\
\hline
\end{tabular}

XXXI. 14./IV. 88. Bei einer curaresirten Katze wurden die vorderen 7. I.enden-, 1., 2., 3., 4. Sacral- und 1. Candalwurzel vom Rückenmark getrennt und ihre peripheren Abschnitte elektrisch gereizt.

11 h. $50 \mathrm{~m}$. Reiz. der 7. Lambalis bei $110-60 \mathrm{~mm} \mathrm{R.} \mathrm{A.} \mathrm{Ruhe.}$

$55 \mathrm{~m} . \quad$, "1. Sacralis

$12 \mathrm{~h}$

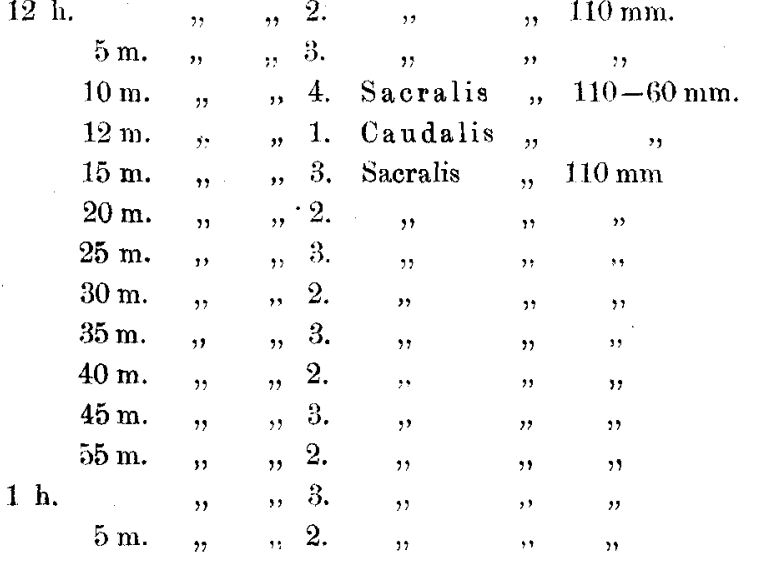

$$
\begin{aligned}
& \text { "Contraction. } \\
& \text { "sehr starke Contr } \\
& \text { "Ruhe. } \\
& \text { " " } \\
& \text { "sehr starke Contr. } \\
& \text { " starke Contraction. } \\
& \text { " sehr starke Contr. } \\
& \text { " starke Contraction. } \\
& \text { " sehr starke Contr. } \\
& \text { "starke Contraction. } \\
& \text { " sehr starke Contr. } \\
& \text { " starke Contraction. } \\
& \text { " sehr starke Contr. } \\
& \text { " starke Contraction. }
\end{aligned}
$$

\begin{tabular}{|c|c|c|c|c|c|c|c|c|}
\hline $47 \mathrm{~m}$ & $"$ & $"$ & 3. & " & $"$ & $"$ & $"$ & $"$ \\
\hline $49 \mathrm{~m}$. & $"$ & , rechten & 2. & ", & $"$ & $"$ & $"$ & Contraction. \\
\hline $51 \mathrm{~m}$. & $"$ & $"$ & 3. & $"$ & ", & $n$ & $"$ & starke Contr. \\
\hline & $"$ & , linken & 2. & $"$ & $"$ & $n$ & $"$ & Contraction. \\
\hline m. & $"$ & $"$ & 3. & , & 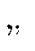 & $"$ & $"$ & starke Contr. \\
\hline $\begin{array}{l}8 \mathrm{~m} . \\
10 \mathrm{~m}\end{array}$ & $"$ & "rechten & $\begin{array}{l}2 . \\
3 .\end{array}$ & $"$ & $"$ & ", & ", & $\begin{array}{l}\text { Contraction. } \\
\text { starke Contr. }\end{array}$ \\
\hline
\end{tabular}

XXXII. 3./X. 88. Bei einer curaresirten Katze wurden auf beiden Seiten die vorderen 2. und 3. Sacralwurzeln vom Rückenmark getrennt und ihre peripheren Abschnitte elektrisch gereizt.

$11 \mathrm{~h} .45 \mathrm{~m}$. Reiz. der linken 2. Sacralis bei $110 \mathrm{~mm}$ R. A. starke Contr. 
Es wurden nun beide Hypogastrici durchschnitten; die elektrische Reizung ihrer peripheren Abschnitte ergah Folgendes.

12 h. $40 \mathrm{~m}$. Reiz. des linken Hypog. bei $110 \mathrm{~mm}$ R. A. Contraction.

$41 \mathrm{~m}$.

, rechten " , , , ,

Es wurden nun die vorderen Sacralwurzeln von Neuem elektrisch gereizt.

12 h. $48 \mathrm{~m}$. Reiz. der linken 2. Sacralis bei $90 \mathrm{~mm}$ R. A. Contraction

\begin{tabular}{|c|c|c|c|c|c|c|c|c|}
\hline $49 \mathrm{~m}$. & $"$ & $"$ rechten & 3. & $"$ & $"$ & $"$ & $"$ & starke Contr. \\
\hline $51 \mathrm{~m}$. & $"$ & " & 3. & $"$ & , & $"$ & " & , \\
\hline $5 \mathrm{~m}$. & ", & " linken & 2. & $"$ & 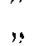 & $n$ & , & $n$ \\
\hline $6 \mathrm{~m}$. & 9 & , & 3. & $"$ & $"$ & $"$ & ", & $"$ \\
\hline $7 \mathrm{~m}$. & $"$ & , rechten & 2. & $"$ & " & $"$ & $"$ & " \\
\hline $8 \mathrm{~m}$. & , &, & 3. & $n$ &, & ", & $"$ & 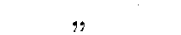 \\
\hline
\end{tabular}

Unsere Versuche zeigen, dass die motorischen Nerven der Blase aus dem Rückenmarke dieses Organ auf zwei Wegen erreichen:

1) Erster - oberer Weg. Rückenmark, 4. und 5. vordere Lumbalwurzeln, rami communicantes, der Lendentheil des Grenzstranges, Nervi mesenterici (superior, medius, inferior), ganglion mesentericum inferins, Nervi hypogastrici, plexus bypogastricus, plexns vesicalis, Blase.

2) Zweiter - unterer Weg. Rückenmark, 2. u. 3. vordere Sacralwurzeln, Nervi sacrales, plexus hypogastricus, plexus vesicalis, Blase.

\section{Frklärung der Abbildung anf 'Tafel VI.}

Die Blasennerven der Katze.

Nn. ad gang. coel. = die Nervenfäden zum ganglion coeliacum.

G. mes. sup. = ganglion mesentericum superius.

$a b=$ Nervenfäden zum ganglion mesentericum inferius.

N. mes. sup.; N. mes. med.; N. mes. inf. = Nervus mesentericus superior, medius, inferior dexter et sinister.

$N$. mes. arteriae $=N$. mesentericus arteriae.

G. mes. inf. = Ganglion mesentericum inferius.

Nn. hypog. = Nervi hypogastrici.

$\mathrm{O}_{\mathrm{s}}$ fem. $=\mathrm{O}_{\mathrm{s}}$ femoris. 
Nh.ad gang coel

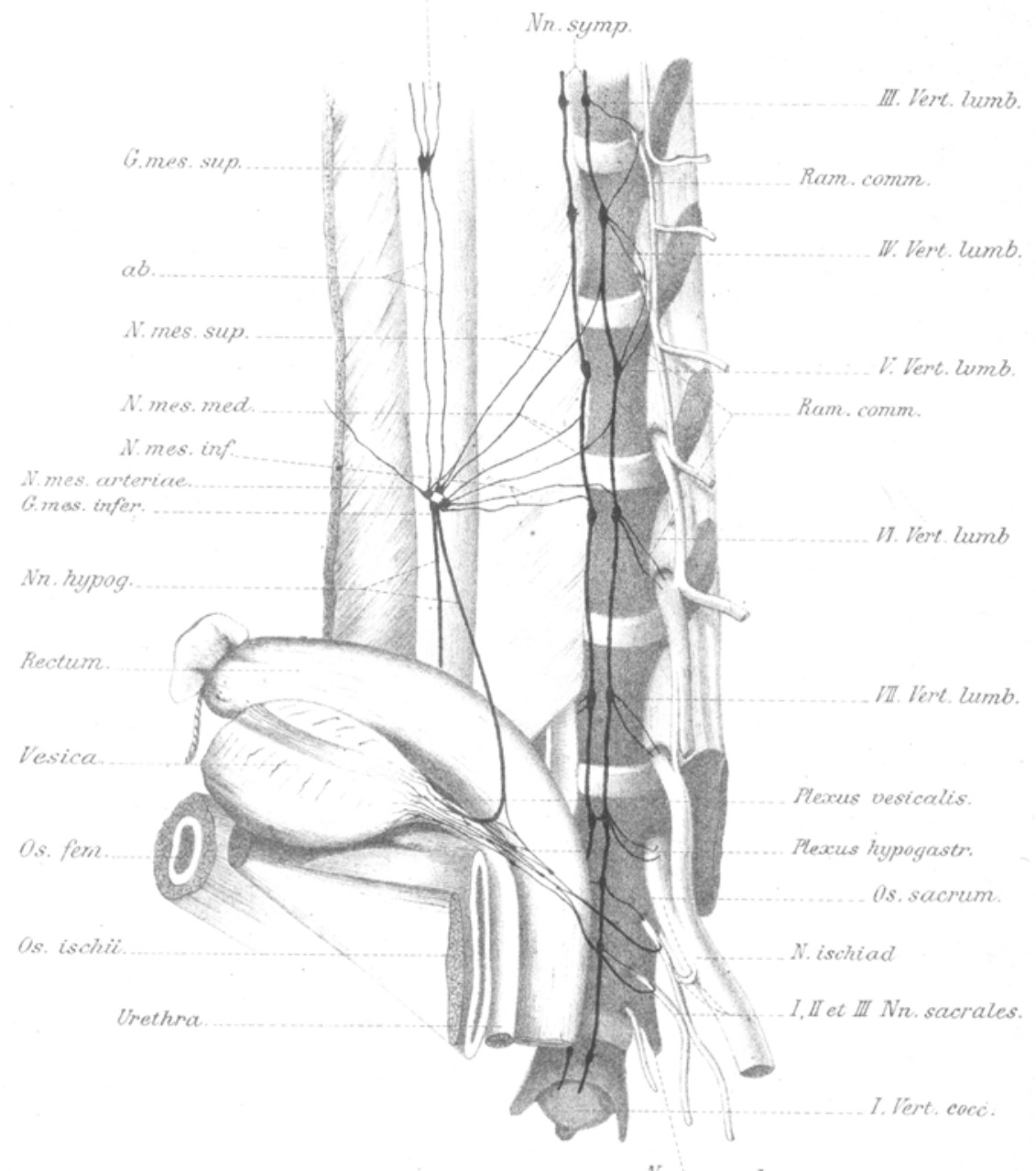

Nnisacrales. 